\title{
La construction de l'image dans la relation sociale
}

\author{
Jacques Defrance
}

\section{OpenEdition}

Journals

Édition électronique

URL : http://journals.openedition.org/trema/2117

DOI : 10.4000/trema. 2117

ISSN : 2107-0997

\section{Éditeur}

Faculté d'Éducation de l'université de Montpellier

\section{Édition imprimée}

Date de publication : 1 décembre 1995

Pagination : 15-34

ISSN : 1167-315X

\section{Référence électronique}

Jacques Defrance, "La construction de l'image dans la relation sociale », Tréma [En ligne], 8 | 1995, mis en ligne le 20 septembre 2013, consulté le 01 mai 2019. URL : http://journals.openedition.org/ trema/2117 ; DOI : 10.4000/trema.2117

Ce document a été généré automatiquement le 1 mai 2019.

Trema 


\title{
La construction de l'image dans la relation sociale
}

\author{
Jacques Defrance
}

\section{Le problème posé : la construction sociale de l'image d'une institution}

1 L'identité de l'éducation physique ne s'est pas ébauchée d'un coup. Le chercheur en histoire s'en rend brusquement compte lorsqu'il tente à de définir l'étendue de son investigation pour analyser l'éducation physique en 1785 ou en 1895 ou en 1996 : l'objet n'a pas toujours de bornes bien précises, c'est-à-dire de définition stricte qui dise où il commence et où il finit. Très vite le chercheur est perdu dans une multiplicité de connexions possibles et de corpus sans fin. Dans la mesure où il s'appuie sur des documents d'époque, le flou de l'image de l'éducation physique est bien celui qui règne en ce temps-là, pour les agents eux-mêmes (même si un brouillage supplémentaire peut résulter de la distance entre l'historien et son objet éloigné).

2 Nous faisons l'hypothèse que l'indétermination n'est pas invariable : à certains moments, les promoteurs des éducations physiques ressentent la nécessité de préciser la définition de ce qu'ils font, de souligner des frontières, d'affiner l'image de la pratique dont ils s'occupent. J'en ai évoqué un exemple à propos de la publication par Georges Hébert du livre Le sport contre l'éducation physique en 1925 (Defrance, 1993), en remarquant que dans ces circonstances les questions de vocabulaire deviennent importantes.

3 On peut se demander dans quelles conditions sociales, propres au champ des activités physiques et des sports, ou plus générales, les agents ressentent-ils le désir de fixer plus précisément les traits de l'institution dont ils s'occupent, d'identifier les lignes de détail de son image? Comment comprendre qu'à certains moments, l'institution fonctionne sur une définition tacite, une doxa, dont le caractère implicite entretien une grande imprécision, ce qui n'empêche nullement son fonctionnement, tandis que dans d'autres conjonctures, il faut indiquer avec soin, quasiment comme des juristes donnant une définition légale, ce qu'il en est de la pratique? 
Il est possible que l'activité de définition des agents ne soit pas le seul facteur qui induit une meilleure précision de l'image de l'institution. Un paramètre sociologique entretient un rapport étroit avec la construction de l'image de l'institution : il s'agit des relations sociales dans lesquelles celle-ci se construit. En effet, l'image d'un objet ne se définit que dans un double système de relations : l'un confronte le sujet connaissant à une expérience du monde sensible, tandis que l'autre engage ce même sujet dans des rapports sociaux. Dans le premier type de rapport, les catégories de perception acquises permettent une aperception de l'objet, tandis que simultanément, l'inclusion de l'individu dans des ensembles sociaux, famille, groupe scolaire ou sportif, profession, classe sociale, etc. lui fournit des critères d'appréciation des percepts qu'il se forme.

L'opération qui permet l'appréciation est essentiellement comparative : c'est un procès de caractérisation et de qualification des objets, qui est toujours relatif. Autrement dit, c'est par rapport à des pratiques "pas excitantes » que d'autres sont «excitantes », par rapport à un athlète médiocre » qu'un autre est "bon", par rapport à une activité plutôt confinée » qu'une autre paraît "naturelle» ou "aérée ", etc. La comparaison est à l'activité cognitive ce que la relation est à l'activité sociale, et l'une s'accomplit par l'autre.

\section{La relation volontaire et les relations structurales constitutives d'un champ}

6 La notion de relation sociale dans le secteur des sports a souvent été illustrée par la relation interindividuelle qui se noue dans un club entre personnes qui ne se connaissaient pas et qui ne se seraient pas rencontrées si elles n'avaient pas adhéré à une organisation sportive. La relation est pensée à travers un exemple d'interaction directe, de connaissance en face-à-face. Or la notion de relation sociale renvoie à autre chose, et le primat accordé à cette variante de rencontre traduit un fantasme, sexuel pour les uns, social pour les autres (une rencontre amoureuse, une relation utile pour la carrière professionnelle), mais toujours libéral, mettant en œuvre les schèmes de l'initiative individuelle et de l'adhésion volontaire, piliers de l'idéologie associative du sport.

7 Il manque à cette sociologie une réflexion sur les relations qui sont structurées durablement, une approche qui évite de les réduire à une dimension interactionnelle, et qui envisage les relations collectives, celles constitutives du champ des activités physiques et des sports : les relations du sportif à l'entraineur, du pratiquant au président de fédération, du débutant au champion, du performer au journaliste, de l'athlète homme à l'athlète femme, de l'éducateur physique au compétiteur sportif, et ainsi de suite. L'ensemble de ces positions, et des relations entre ces positions, définit un état donné du champ, et il structure i.e. préforme, contraint, oriente, balise, détermine plus ou moins étroitement - les face-à-face qui s'y développent.

\section{Lier, délier : dépendance et autonomie}

Un certain nombre de travaux sociologiques et historiques de ces dernières années ont contribué à montrer la nature relationnelle des phénomènes sociaux et ce programme a été suivi dans le secteur des éducations physiques et des sports. Les liens entre le patriotisme, puis le nationalisme, et les gymnastiques et les sports (notamment au tournant du XIXe et du XXe siècle), les relations entre les activités physiques et l'école, 
l'armée, la médecine ou l'économie ont fait l'objet de travaux. Contre une vision de spécialiste qui isole le sport ou l'éducation physique au point d'en faire une entité apparemment mue par une «logique interne », l'approche par les sciences sociales n'a cessé de "contextualiser", et de "replacer dans les conditions de sa production", l'institution sportive et l'éducation physique. Insistons sur les apports de ce programme: il montre comment le contenu même des pratiques est affecté par les normes des milieux qui les organisent (programme d'exercice pour les femmes, choix de méthodes d'entraînement, etc.), il souligne les liens entre la culture des groupes impliqués et la pédagogie retenue pour initier ou perfectionner (les manières d'enseigner des militaires, les oppositions entre théoriciens et praticiens, etc.), il révèle enfin les stratégies d'alliance entre divers groupes qui contribuent à associer gymnastes ou sportifs à des « autorités diverses, médecins et hommes de science, pédagogues et hommes politiques, vedettes et stars issues de divers univers, permettant aux premiers de recevoir un peu de légitimité et à leurs activités d'obtenir une reconnaissance un peu élargie. Une série de travaux a même été jusqu'à promouvoir la sociabilité comme dimension privilégiée de l'activité «sportive » ordinaire («sportif» entendu au sens large), la décrivant comme un moyen de créer du lien, et de renforcer des relations interindividuelles. Bref, l'objectif a été de faire comprendre la construction de l'institution «sportive » en donnant une idée de sa "fabrication" par ses promoteurs et par ses membres au moyen de relations et de liens divers.

9 J'aimerais poursuivre cette entreprise en montrant que l'institution s'est aussi construite en défaisant des liens, en rompant des relations, en se dégageant de "loyautés" premières qui, initialement, attachaient l'exercice physique à d'autres pratiques et à des valeurs « autres » que celles du monde » sportif ». Autrement dit. un noyau relativement homogène de pratique se sépare lentement de tout un ensemble d'activités qui venaient en complément, en préalable, en appui : en effet, jusque tard dans le $\mathrm{XX}^{\mathrm{e}}$ siècle, certains n'imaginent pas un rassemblement de jeunes gymnastes sans un banquet, un défilé, un comité de patronage qui mêlent les aînés et ces jeunes; les éducateurs physiques ne conçoivent pas une formation de l'adolescent qui sépare le sport des jeux et des évolutions collectives; les revues de "sports " à vocation générale ne peuvent traiter de sports athlétiques, sans tourisme automobile, chasse à courre et culture physique d'entretien, etc. Bref, la démarcation entre ce qui est du («vrai ») sport et ce qui n'en est pas ne se précise et ne se renforce que lentement. La netteté de cette frontière est un indicateur de l'autonomisation du champ sportif, processus sur lequel je travaille (Defrance, 1995). 
Avant le Lauwn-tennis, la Paume. Le jeu royal, c'est le jeu de paume. Jeu de paume français. Époque de Louis XIV.

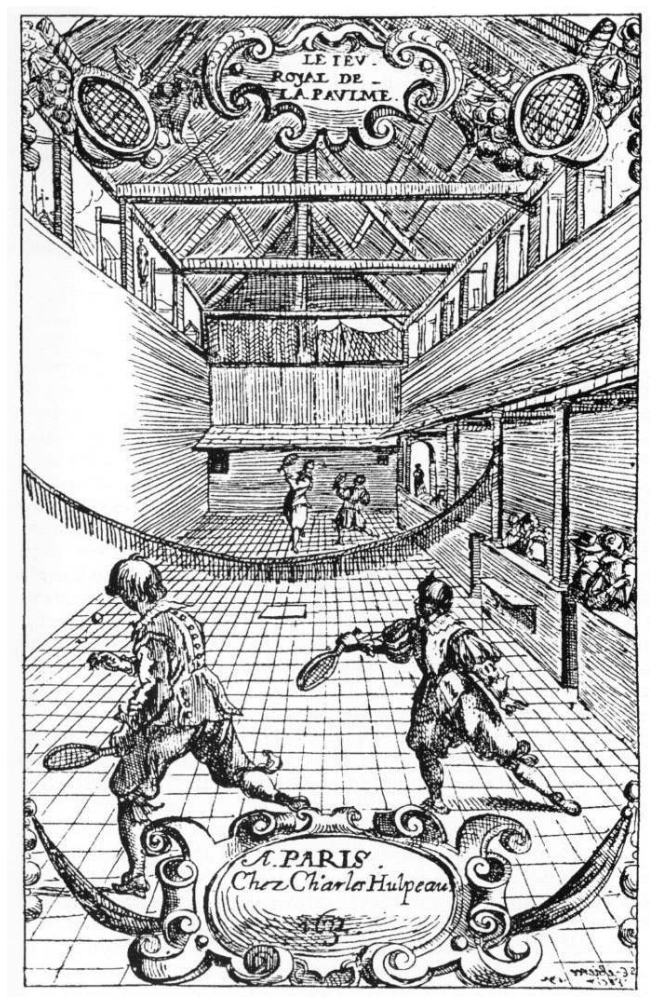

Académie des sports et Comité national des sports, Encyclopédie des sports, Tome I, Paris, Librairie de France, 1924

\section{Un aspect négligé par les chercheurs}

10 La construction du champ par la décomposition du lien est peu abordée. La désaffection pour ce sujet d'étude révèle un postulat courant parmi les chercheurs. Ils conservent une vision optimiste, constructive de l'histoire de l'institution, qui est implicitement individuelle. Ils voient l'établissement d'une institution, par exemple le « sport », comme une œuvre partant d'une idée personnelle, propre à un individu (Pierre de Coubertin) ou à quelques individus, qui tissent petit à petit des liens, qui installent des mécanismes de régulation, qui forgent des règles collectives, enfin qui enclenchent une gestion rationnelle appuyée sur un appareil administratif («bureaucratisation»), si bien que les recherches limitent leur attention à la construction de relations, au montage de dispositifs collectifs partant d'«initiatives individuelles». En somme, les analyses s'appuient sur une perception proche du sens commun qui fait en général apercevoir l'état des choses d'autrefois comme étant plus « simple », plus «spontané », plus proche des individualités, et l'état des choses actuel comme étant plus "socialisé », plus "règlementé », plus imbriqué clans de multiples contraintes (économiques, politiques, éducatives). Or, s'il est certain que des mécanismes nouveaux sont mis en place à mesure qu'une institution s'installe, croit et se complexifie, il resterait à prouver qu'au départ il n'y en a pas, et que dans les premiers temps du « sport » nous avons affaire à des unités libres, des individualités encore non liées. 
11 Ce point fait question : le passé est un lieu de projection privilégié, et on ne peut le qualifier sans une enquête. Il est en général traité à partir d'une vision individualisante qui s'appuie sur quelques témoignages rétrospectifs nostalgiques (des retraités qui évoquent l'époque de leurs vingt ans). Mais que dire de l'extrême implication des militaires et des patriotes dans la gymnastique, le tir, l'athlétisme, l'alpinisme, de 1880 à 1920-1940, ou de l'omniprésence des éducateurs et des pasteurs dans le sport des origines au sein des public schools anglaises du XIX ${ }^{\mathrm{e}}$ siècle ? Les vigoureux gymnastes et les jeunes sportsmen étaient-ils des « électrons libres » à la recherche d'une place dans la société ? ou bien au contraire, n'étaient-ils pas des individus très précisément inscrits dans des milieux sociaux, des positions et dans des institutions éducatives et politiques, mais «non sportives » ? Pour restituer fidèlement les phénomènes, il y a lieu de mener des enquêtes sur les relations de l'institution gymnastico-sportive à d'autres secteurs de la vie sociale, avec une égale absence de préjugés et avec une méthode uniforme, pour la fin du XIX siècle comme pour la fin du $\mathrm{XX}^{\mathrm{e}}$ siècle.

Un second facteur explique l'absence des mécanismes de rupture de relations dans la description du développement des institutions "sportives». En effet, il s'agit ici du versant négatif de la genèse institutionnelle (du moment négatif, dirait Hegel). Son analyse suppose de se concentrer sur des aspects déclinants, sur des liens qui se défont, mais aussi sur ceux qui étaient partie prenante de ces relations et qui les voient s'évanouir très souvent à leur corps défendant : bref, il s'agit de s'intéresser à des groupes perdants, à des pratiques qui tombent en désuétude, ce qui souvent signifie des agents vieillissant, qui se replient ou se reconvertissent, des entités qui perdent de l'importance, des images qui se ternissent, etc.

Or, l'étude du sport à travers les sciences sociales est restée pour beaucoup une quête de légitimité pour leur discipline et partant pour eux-mêmes : cette quête n'atteint son but que lorsqu'on transforme l'histoire des «sports » en success story, en tableau montrant des progressions, des constructions, des «acquis», bref une accumulation. Que la construction, la transformation, la reproduction, la réorientation d'une institution laisse un certain nombre d'agents ou de groupes d'agents «sur le carreau » n'est pourtant pas une idée très originale: elle paraît banale en économie avec le déclin des groupes attachés à des formes d'activité traditionnelles supplantées par de nouvelles (les artisans face à l'industrie manufacturière, les petits commerçants face à la grande distribution, les agriculteurs sur des exploitations familiales, etc.), mais on sait aussi que d'autres institutions, en changeant, ont conduit à la marginalisation de divers groupes sociaux (l'expansion de la médecine réduit l'influence des guérisseurs, l'accroissement de la formation technique scolaire éclipse l'apprentissage artisanal, etc.).

\section{Questions de méthode}

\section{Les phases d'autonomisation}

14 Les moments de déclin de certains types de relations nouées autour des activités physiques et des sports sont repérables par quelques signes empiriques : à partir de ceuxci, on construit des projets d'enquête. Parmi ces signes, l'un est inscrit dans la trajectoire biographique des agents qui fuient hors des positions dont le statut décline. Au plan des grandes masses d'affiliations, il s'agira de mesurer la décroissance des effectifs dans une forme de pratique organisée. Ou encore, on pourra repérer, à un niveau plus individuel, 
des « sorties » hors du champ des activités physiques et des sports, des abandons » ou des « reconversions».

Trois moments identifiés après examen documentaire paraissent relever de cette problématique.

- Vers 1895 d'abord, on enregistre un mouvement de reclassement parmi les promoteurs du sport, avec abandon ou retrait de la scène de la part de certains d'entre eux, alors que dans les années 1880 c'était un mouvement d'attraction inverse que l'on enregistrait : comment en rendre compte?

- Dans les années qui suivent la première guerre mondiale, ce sont les liens entre l'éducation physique et la préparation militaire qui se défont:

- Un autre moment est repérable, qui signale une décomposition de lien plus lente : entre les années 1950 et les années 1960, la prise en considération d'un réseau de promoteurs « pro sportifs » par l'Etat se fait au prix d'une rupture des relations avec un autre réseau de promoteurs à orientation " pédagogico-hygiénique ».

L'objectif de la présente étude n'est pas d'assimiler ces moments, mais plutôt d'examiner avec quelles catégories d'analyse - catégories sociologiques descriptives et explicativeson peut aborder des moments de la dynamique du champ au cours duquel des agents sont évincés, des pratiques sont déqualifiées, et des "courants " sont marginalisés, ce qui conduit à terme à une recomposition du champ (un nouvel « état »).

La re-configuration de cet espace équivaut à un réaménagement des relations qui s'y nouent, tandis que change l'image des diverses catégories d'activités qu'on y pratique.

\section{Une sociologie de la critique}

L'analyse historique du déclin de la légitimité d'une pratique et de la décomposition de liens structurels sous-jacents suppose de définir comment on utilise les documents « critiques » que l'on récolte.

Soit un exemple d'analyse évoquant le dénigrement de la gymnastique :

La gymnastique toute entière, écrit E, Pécaut "reste chose morte", stérile, propre à faire bonne figure dans les règlements et les programmes, voire dans les expositions nationales ou internationales, mais en réalité impuissante... à inspirer le goût de l'activité physique ». En opposition absolue avec ce formalisme physique, "la gymnastique anglaise ou sport» (Tissié)... va donner aux adolescents « une enivrante impression de liberté » (Blanchard).

20 Jacques Thibault construit son analyse du développement des sports qui repousse la gymnastique vers la marge à partir de ces déclarations (Thibault, 1972, pp. 75-76). Elles sont prises pour argent comptant, i.e. que l'historien adopte le point de vue de celui dont les propos sont cités : il s'en sert cent ans plus tard pour expliquer les raisons du déclin de cette éducation physique "stérile» et son remplacement par quelque chose de plus « intéressant ».

21 La démarche proposée ici va dans un sens différent: ce n'est pas parce que les pratiques sont, dans leur substance même, "mauvaises", "ennuyeuses", que des critiques apparaissent, que l'image est ternie, ce qui agirait sur les agents qui l'abandonnent ; c'est, à l'inverse, parce que des agents peuvent établir des positions concurrentes à cette gymnastique, parce qu'ils peuvent trouver autre chose et "sortir », parce qu'ils peuvent comparer, ou parce que les rapports de forces sont en train de pencher pour d'autres 
pratiques (par exemple le sport plutôt que la gymnastique), que ces pratiques sont décrites sous leurs aspects négatifs et que leur image est noircie.

Les propos d'Élie Pécaut en 1882 pourraient être éclairés - leur signification pourrait être dégagée - en les replaçant dans l'argumentation critique générale que cet homme politique adresse à l'école, en précisant quelle position il occupe; de même il faudrait indiquer qu'à cette même date, des agents - mais ils diffèrent de Pécaut - trouvent encore un intérêt dans la gymnastique, que cette pratique progressera encore jusqu'au $\mathrm{XX}^{\mathrm{e}}$ siècle, etc. La question est alors de rendre compte du fait qu'au même moment, une même pratique a une image excitante pour certains, et une image terne pour d'autres.

\section{Critique ordinaire et signe de déclin}

Pour l'analyste, il est nécessaire de discerner :

- le dénigrement qui prend place dans une structure de relations concurrentielles stables et qui signale la position extérieure et antagoniste de son auteur au sein d'une différentiation sociale donnée : par exemple, Vincent de Laprade disant du mal de la gymnastique disciplinée des lycées publics dans les années 1870, parce qu'il est d'un milieu social qui se sent extérieur et supérieur et qui privilégie d'autres activités corporelles : ceci prenant place dans une situation où la gymnastique se développe lentement :

- et le dénigrement qui est un indice de la décomposition d'un lien et de la dé-légitimation d'une pratique et du groupe qui la portait. Ce noircissement atteint alors une pratique en voie d'abandon, et il est porté et amplifié par tous ceux qui abandonnent : il vise des principes qui perdent de leur pertinence, tel le principe qui voudrait que l'activité physique soit précisément surveillée et dosée par un adulte doté d'un sens moral quand un groupes d'enfants s'exerce.

La différence entre ces indices se lira de deux façons. D'une part dans le texte lui-même, dans le document, où le choix des mots indiquera le degré de réprobation et l'avancement de la disqualification de la pratique visée. D'autre part dans la situation où se trouvent les protagonistes, au moyen d'informations sociologiques complémentaires qui viendront qualifier la position de ceux qui emploient chaque terme, chaque argument qui a pour effet de noircir le tableau : c'est ici que les trajectoires des agents sont restituées, et que l'histoire sociale des positions est combinée avec l'histoire des prises de position (c'est-àdire une forme d'histoire des idées). 
Le «Desports » chez nous aïeux. Jeu de ballon et tir à la butte. Gravure française du seizième siècle.

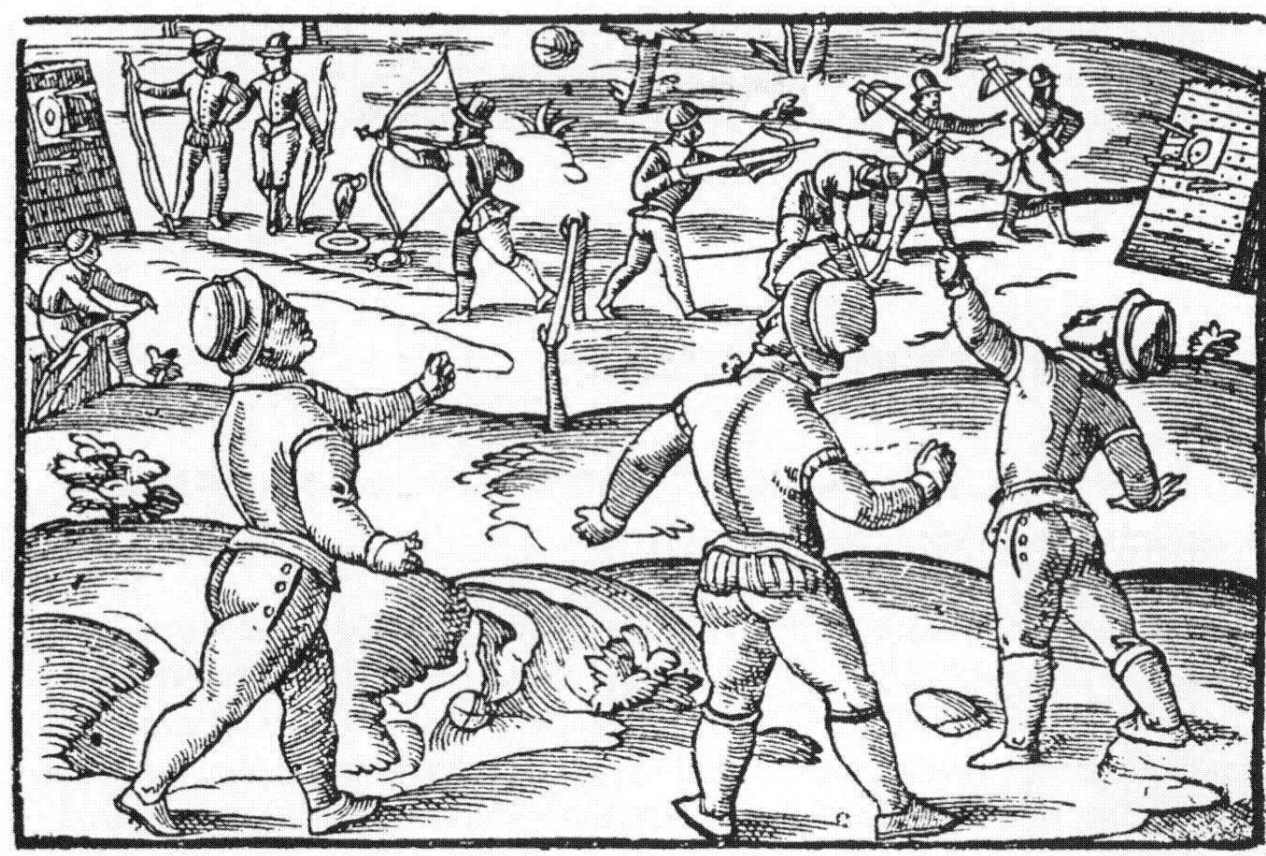

Jusserand. Les sports et Jeux d'exercice de l'ancienne France, Plon, cité in : Académie des sports et Comité national des sports, Encyclopédie des sports, Tome I, Paris, Librairie de France, 1924.

Avant le football la soule. Début d'une partie de soule (football), en Bretagne, dix-neuvième siècle. Soule, choule ou cholle, c'est un des plus anciens jeux de la vieille France. 


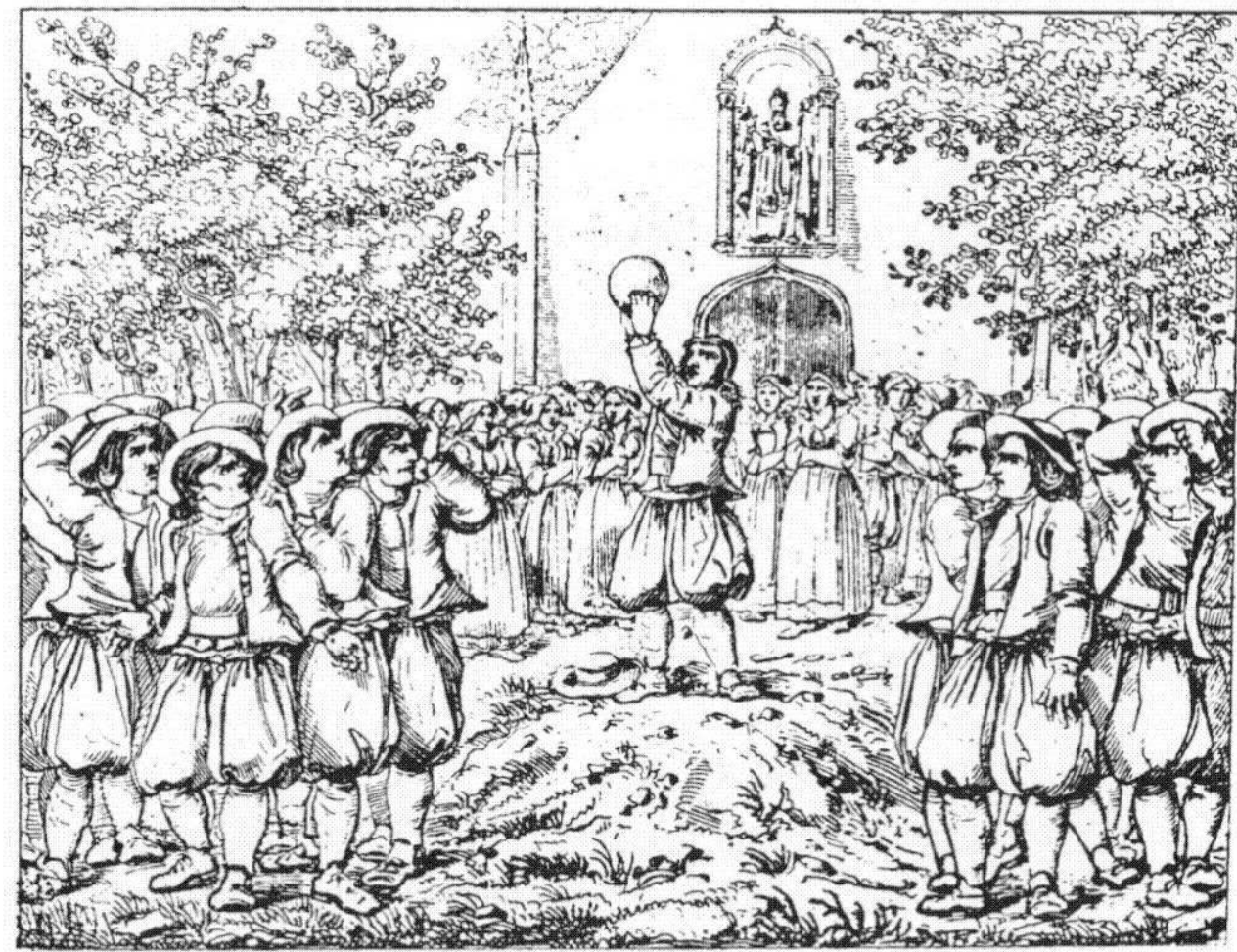

Jusserand. Les sports et Jeux d'exercice de l'ancienne France, Plon, cité in : Académie des sports et Comité national des sports, Encyclopédie des sports, Tome I, Paris, Librairie de France, 1924.

\section{Analyses socio-historiques}

\subsection{L'abandon du projet de lier le sport à l'éducation physique scolaire vers 1895}

Dans les années 1880, le champ des activités physiques et des sports, fraîchement constitué, connaît une dynamique remarquable sous l'effet d'une pression du champ politique alors dominé par les Républicains. Dans la lancée des lois sur l'école et l'armée, l'éducation physique gymnastique et militaire reçoit une impulsion notable, tandis que les sports compétitifs démarrent (Defrance, 1987 ; Arnaud, 1991 ; Arnaud, Camy, 1986).

Cette conjoncture du milieu des années 1880, qui permet à la gymnastique d'être « portée » par l'école et par l'armée, renforce la perspective d'appuyer le développement des sports sur cette même institution scolaire. Une perspective d'action, c'est une voie aperçue comme relevant de l'ordre du possible et qui semble, dans des circonstances particulières, être à portée, accessible. Dès qu'elle se dessine et préfigure des chances de réussite, les agents qui l'adoptent se multiplient.

Ainsi, vers 1887-88, les promoteurs des activités physiques prétendant installer diverses sortes de pratiques d'exercice dans l'enseignement sont tous sur le même enjeu :

- Georges Démeny qui s'appuie sur la gymnastique instituée et le corps des professeurs dotés du Certificat d'aptitude à l'enseignement de la Gymnastique, et propose une amélioration du système de préparation des enseignants (fondant le Cercle de Gymnastique Rationnelle, etc. : cf. Pociello, 1972) ; 
- Philippe Tissié qui projette de faire entrer les jeux et les sports et fonde la Ligue Girondine d'Éducation Physique ;

- Paschal Grousset dont l'objectif est assez proche de celui de Tissié, avec la Ligue Nationale d'Éducation Physique ;

- Pierre de Coubertin qui, avec le Comité pour la Propagation des Exercices Physiques dans l'Éducation, projette de rallier les enseignants à sa cause « sportive » et de pénétrer le milieu de l'enseignement secondaire ;

- et, proche de lui, Gorges de Saint Clair, qui, avec l'USFSA, vise pendant un temps à développer le ferment sportif sur le terrain scolaire, avec les associations sportives scolaires.

Dans cette conjoncture, les sports et les gymnastiques sont fortement " pressés de faire corps avec les objectifs éducatifs de l'école : on leur impute (processus d'attribution) une fonction dans la formation patriotique des jeunes gens, ou encore dans l'équilibre de vie des élèves (voir le débat sur le surmenage des années 1880: Arnaud, in Pociello, dir., 1985). La foi dans la fonction transformatrice de l'école est à son comble dans le champ politique et social (Furet, Ozouf, 1977).

Dix ans plus tard, vers 1894-96, le faisceau de relations nouées autour de l'institution scolaire va se défaire ou se relâcher. L'enjeu scolaire étant dévalué, les promoteurs des activités physiques et des sports réorientent leur action, tout en détachant en grande partie la question du développement de leurs activités de la question scolaire et de l'éducation physique. Les trajectoires individuelles enregistrent ce changement de conjoncture collective, si bien qu'elles prennent des infléchissements parallèles, à savoir ;

- de Saint Clair est évincé des instances du sport par de Coubertin en 1894 et retourne à la diplomatie :

- de Coubertin s'oriente vers l'internationalisation du sport et l'organisation de Jeux olympiques (congrès en 1894), préférant désormais que le sport s'organise en clubs civils plutôt qu'en associations scolaires : dès lors, le développement pour les premiers sera plus rapide que pour les seconds (Le Roux, in Pociello, dir., 1985) ;

- Tissié reste un peu à l'écart avant de partir en Suède et de se convertir à la méthode « suédoise « (Ling) ;

- Grousset abandonne sa Ligue nationale pour se tourner vers la politique :

- enfin, Démeny connaît une période de retrait, suite à ses démêlés avec Marey (fin 1893-1894) (Pociello, 1972).

Pendant un temps, l'éducation physique et le sport vont se délier relativement. Après avoir eu un congrès commun à l'Exposition Universelle de 1889, « pour la propagation des exercices physiques dans l'éducation » (avec de Coubertin, de Saint-Clair, pour le sport, à côté des gymnastes Sansbœuf, Blatin, Féry d'Esclands, etc.) ${ }^{1}$, chacun organise son propre congrès : le congrès national d'Éducation physique à Bordeaux en 1893 ("deuxième ", après un congrès à la Sorbonne à paris en 1892), et le congrès constitutif du projet des Jeux olympiques (sportifs) à Paris en 1894.

À la phase où sports et éducation physique sont proches (entre 1885 et 1894) correspond l'usage d'un terme de vocabulaire qui permet d'englober l'ensemble des pratiques rassemblées : "l'exercice physique ». Il y a même recours au concept de "mouvement " (par ex.: Dupuy, 1893; Marey, 1894). Un «Comité des Exercices Physiques dans l'Éducation» publie un manuel de jeux et d'exercices en 1889. Le docteur Monin argumente en faveur de «la santé par l'exercice» en 1889, tandis que Lagrange publie trois ouvrages sur "l'exercice» entre 1890 et 1894, etc. (Monin, 1889; Lagrange, 1890,1891, 1894 ; voir aussi : Bonnefont, 1890 ; Couvreur, 1890 ; Séhé, Strehly, 1890). Ce 
n'est que dans les années 1890-1900, que le terme éducation physique » va remplacer "gymnastique», "exercice», et d'autres appellations ("gymnastique rationnelle», «jeux et exercices ») (Bernard, Vigarello, Pociello, 1975). Parallèlement, l'acception du mot «sport» doit se préciser elle aussi, à mesure que les jeux sportifs des débuts se codifient et s'appuient plus directement sur un système de compétitions. Une image plus claire et séparée du sport et de l'éducation physique se dessine (par exemple in Lagrange, 1892).

Tableau 1. Nombre de publications en éducation physique, gymnastique, culture physique (les sports étant partiellement pris en compte).

\begin{tabular}{lcc} 
& $\begin{array}{c}\text { Total : } \\
\text { gymnastique scolaire, } \\
\text { bataillons scolaires } \\
\text { anatomie appliquée } \\
\text { à l'E.P., sports, etc. }\end{array}$ & $\begin{array}{c}\text { Dont: } \\
\text { gymnastique } \\
\text { suédoise }\end{array}$ \\
Périodes & 53 & 1 \\
$1885-1889$ & 43 & 3 \\
$1890-1894$ & 35 & 11 \\
$1895-1899$ & 53 & 11 \\
\hline
\end{tabular}

Mieux discernée, l'éducation physique peut entrer dans une phase d'élaboration technique : en particulier, on assiste à l'importation de la méthode suédoise après 1895, avec une multiplication des traductions des œuvres des maitres de Suède ou des pays Scandinaves (tabl. 1: la méthode existait bien auparavant, et avait fait l'objet de tentatives d'importation depuis le milieu du XIX siècle. Démeny effectue une mission en Suède en 1891 et en rend compte en 1892 : Tissié en parle en 1893, et effectue une mission en 1898). Puis débuteront, vers 1905, les réflexions méthodologiques d'Hébert (méthode naturelle) et de Démeny (version « mouvement complet, continu et arrondi »).

D'un champ des activités physiques et des sports polarisé par des enjeux construits autour du système scolaire (vers 1885), on passe à un autre état du champ (vers 1896). Tout se passe comme si les pratiques d'exercice subissaient un double processus, d'internationalisation (olympisme, rencontres bilatérales et diffusion de la métropole vers les colonies) et de différenciation (avec l'établissement de diverses formes de pratiques), au moment où s'affirment les enjeux géopolitiques qui vont mener à la guerre de 14-18 et où émerge le mouvement ouvrier dans son organisation moderne (partis, syndicats, formes de mobilisation, etc.).

\subsection{La suppression des liens entre éducation physique et préparation militaire dans les années 1920}

L'éducation physique à base gymnastique a été liée, tout au long du XIX ${ }^{e}$ siècle, à l'objectif civique de former des citoyens vigoureux, bons défenseurs de la patrie. La construction est politique et intéresse autant les civils que les militaires (Defrance, 1987). Durant les dernières décennies du XIXe siècle, la connexion est encore renforcée sous l'influence des nationalistes, et la leçon de gymnastique prend une allure militaire (notamment avec 
l'épisode des «bataillons scolaires ", entre 1882 et 1889). Des relations sont établies entre la formation scolaire et la préparation militaire des jeunes gens, si bien que l'image de l'éducation physique est confusément associée à celle de défilés, d'uniformes et d'ordre criés par des sous-officiers.

Or, après la guerre de 1914-18, sous l'effet de plusieurs facteurs, l'idée d'une préparation militaire de la population décline (Spivak, 1983). Par contrecoup, la pression exercée par les militaires sur l'éducation physique décroît, et le Ministère de la Guerre cède la gestion de l'éducation physique au Ministère de l'Instruction Publique vers 1927-29. L'école de Joinville entre dans un déclin progressif à mesure que les IREP se mettent en place, après 1927 (Spivak, in Arnaud éd., 1981). Ce mouvement se traduit dans la biographie de personnages comme Maurice Boigey qui, fortement impliqué dans l'éducation physique de la jeunesse et la recherche de bases scientifiques pour la conduire, va finalement quitter l'armée et se tourner vers la médecine thermale et le régime de vie du troisième âge (Favriou, in Andrieu, éd., 1989).

L'éducation physique va changer d'image dès lors qu'elle se "civilise ", c'est-à-dire qu'elle perd petit à petit ses attributs militaires et patriotiques. Elle devient un enjeu d'affrontements entre médecins et éducateurs, tandis que l'organisation et la perception de la pratique s'individualisent partiellement. Face à une pratique sportive qui prend une forme de loisir compétitif valorisant les individualités, l'éducation physique aura à se définir et à opérer des changements, soit vers une approche plus " pédagogique » prenant en compte la psychologie de l'élève (dans les années 1930, avec Van Schagen, 1933; Lafarge et Nayrac, 1934 ; etc.), soit vers une approche clinique (travaux des IREP et des médecins : par exemple, Tissié, 1919).

\subsection{La relégation par l'État des éducateurs physiques partisans d'une doctrine d'éducation en sport (années 1955-1965)}

Une autre transformation des relations dans le champ "sportif» affecte l'image de l'éducation physique. Dans les années 1950, les éducateurs physiques groupés autour des méthodes naturelle, suédoise ou autre, attendent une politique qui décide d'une orientation ferme dans le sens qu'ils souhaitent en matière de sport. Lorsque, au début des années 1960, les rouages organisationnels créés par les gaullistes se mettent en place, ils viennent participer aux commissions et expriment leurs positions, pressentant que les grandes décisions vont être prises.

Le groupe des éducateurs physiques est en grande partie rassemblé dans un organe fédératif - le Comité National de l'Éducation Physique - qui fait en quelque sorte le pendant du Comité National des Sports. Il a par ailleurs des appuis extérieurs, des alliés, qui partagent ses vues et les expriment dans des cercles élargis : par exemple, JeanFrançois Brisson, journaliste au Figaro. Lorsque les commissions sont créées au Haut Commissariat à la Jeunesse et aux Sports pour aider Maurice Herzog dans sa politique, les éducateurs physiques et leurs alliés peuvent y figurer. Il y a 15 commissions spécialisées qui s'ajoutent au Comité National des Sports (CNS) que le Gouvernement ranime.

À la séance inaugurale du C.N.S., Brisson lit une note intitulée «Élaborer une politique sportive », manifestant bien l'intention des personnes invitées de ne pas laisser l'État seul dans le rôle de conduire les affaires sportives: il approuve le projet de stimuler le développement du sport, tout en ajoutant une citation de Giraudoux : «L'éducateur public 
doit donner au sport ses règles morales ». Et dans les attributions du colonel Crespin, qui sera la cheville ouvrière de la politique sportive, il demande d'ajouter : son action devra être " strictement conforme dans l'esprit et dans la lettre, aux directives générales données en matière d'amateurisme par le Comité International Olympique». Ces demandes sont approuvées par Herzog en ce 11 avril 1961.

Or le Haut Commissariat prend une orientation « sportive » compétitive vers 1962-64, qui va complètement diverger de ces principes. Il va " couper les ponts » avec le réseau des éducateurs physiques partisans d'une doctrine d'éducation, de façon assez brusque, mais sans le déclarer ouvertement (par analogie, on pense à la façon dont de Gaulle se convertit à la politique de l'indépendance de l'Algérie).

41 Le programme de contrôle du sport par l'éducateur s'accompagne de l'application de la doctrine amateuriste du C.I.O.. Ils gardent des soutiens, par exemple au sein de la commission de la Doctrine, présidée par Jean Borotra. Mais cette commission, installée par le Haut Commissaire en 1961, n'est plus dans son orientation lorsqu'elle achève ses travaux en 1965. Ses conclusions reçoivent un accueil froid au sein des services administratifs les plus engagés dans la politique du moment ${ }^{2}$. Elles seront publiées dans un rapport dont l'écho sera assez large (Essai de Doctrine du Sport, 1965), mais qui sera la dernière manifestation d'envergure d'une conception éducative du sport placée sous l'autorité des éducateurs (et des éducateurs physiques).

Que devient le Comité National de l'Éducation Physique qui soutient ce programme ? Créé en 1961, et il est dissous en 1973, « son objectif étant atteint » selon un document officiel ${ }^{3}$. Une analyse de quelques incidents » survenant dans les années 1965-70 peut montrer comment les membres du C.N.E.P. sont marginalisés par les décideurs du Ministère Herzog, et comment leur prétention à contrôler le sport est brusquement réfutée ${ }^{4}$, Sa philosophie de l'éducation continue de s'exprimer, mais il faudrait étudier les lieux où elle trouve une tribune ${ }^{5}$. Il faudrait se pencher sur les stratégies de reconversion ou de repli que les membres du C.N.E.P. sont contraints d'engager (par exemple, l'abandon de l'éducation corporelle des enfants scolarisés pour celle des adultes, avec la "gymnastique volontaire », la « gymnastique utilitaire » ou le » sport corporatif»).

Dans cette conjoncture, les traits accolés à l'ancienne éducation physique (suédoise, naturelle, éclectique, construite) ne sont pas seulement des attributs négatifs d'usage banal dans la polémique ordinaire entre courants concurrents d'E.P. : il se développe une argumentation disqualifiante beaucoup plus cohérente et permanente, dans laquelle les « sciences » viennent apporter leur concours. Il s'agit en quelque sorte d'« objectiver » le caractère « inférieur » ou " dépassé » des vieilles méthodes : ce sera le sens de quelques expériences armées de ressources "scientifiques», par exemple celles de Le Boulch avec ses références psychologiques et physiologiques. Peut-être faut-il aussi compter avec l'entrée en scène des historiens et des sociologues qui viennent montrer que certaines méthodes d'éducation physique " appartiennent au passé », ou que les activités sportives sont des « pratiques d'avenir » et répondent à une demande sociale croissante (travaux de Ulmann et de Dumazedier, puis d'autres).

Dans le cours de cette poussée d'autonomisation, quand des liens se défont entre une forme d'éducation physique sportive et une forme plus traditionnelle attachée à des normes éducatives, l'image de la première s'affine et est valorisée, tandis que la seconde est définitivement reléguée au rayon des pièces de musée. L'éducation physique ne se réfère plus à une doctrine d'éducation, et son image se rapproche de celle des techniques sportives. 


\section{Conclusions} disposent. Ainsi, dans les années 1910-1920, lorsque la vision taylorienne du travail ouvrier se diffuse, les perceptions collectives du geste gymnastique, du travail culturiste, de l'entraînement sportif, sont modifiées. La classification des formes de motricité que programme l'éducation physique est réaménagée et, peut-être, affinée. Mais elle est simultanément soumise à un champ de forces, celui des relations sociales, et 
particulièrement à des mouvements d'adhésion enthousiastes chez des patrons, des médecins, des hommes politiques, au modèle d'usage rationnel du corps qu'est le travail taylorisé, auxquels s'opposent des mouvements de répulsion aussi forts, face à une activité perçue comme automatisme, exécution mécanique et réduction de l'individu au statut de rouage asservi à un système.

Ce sont de nouveaux liens de l'employeur à sa main d'œuvre qui se créent quand un processus de travail est taylorisé. Ces sont aussi de nouvelles formes de relations qui s'établissent lorsque l'éducateur physique ne doit plus s'identifier à l'éducateur patriote de l'école Républicaine, ne doit plus agir sur la base d'un savoir-faire militaire, ou ne doit plus maintenir un écart entre son action pédagogique et les pratiques sportives que ses élèves connaissent par ailleurs dans leur vie sociale (famille, loisirs). Les frontières se déplacent : après avoir soigneusement marqué les différences entre éducation physique et sport (de 1880 à 1960 : spécialement Hébert, 1925) et surveillé scrupuleusement comment les enfants passaient la frontière, les deux fusionnent dans les années $1960 \mathrm{au}$ point que les enseignants chargés de cette matière reçoivent le nom de " prof, de sport ». Les distances perçues entre pratiques sont un bon indice empirique de la distance sociale entre les pratiquants. J'ai montré comment, lorsque la distance est extrême, et qu'un groupe efface de son horizon social un autre groupe, la pratique de l'autre ne reçoit plus le nom qu'elle pourrait partager avec la pratique des premiers : ainsi, le nom même de "gymnastique» ("gymnase ») est refusé aux pratiques de force et d'acrobatie par les représentants de la forme légitime de gymnastique du XIXe siècle (Defrance, 1987). De façon comparable, l'idée que la course à pied sur route puisse relever de l'athlétisme véritable dans le courant des années 1970 est rejetée, repoussant cette pratique vers une catégorie à part (Defrance, in Pociello, dir., 1985).

Le pont entre les relations sociales et les relations logiques, les structures sociales et les structures mentales, ne s'établit que si l'on pose le problème sociologique en évitant le cognitivisme pur (Brunner, 1991) : le processus de connaissance n'est jamais un processus de pure perception rationnelle et sereine dans lequel il s'agirait d'identifier, de qualifier et de classer des objets, donc de façonner simplement des images. Il est d'entrée de jeu un processus pratique et sensible, et la connaissance est une forme d'engagement dans le réel, une sensibilité et une attraction dirigée, complétée par des répulsions elles aussi bien localisées (ce que montre Merleau-Ponty : voir Ostrow, 1990). En ce sens, le travail de Bourdieu sur les catégories de jugement, du goût et du dégoût, et leur structuration sociale, est décisif (Bourdieu, 1979 et 1980) : il objective comment l'activité connaissante et classificatrice engage des dispositions acquises, un habitus, qui ont une forme relationnelle, penchant, attraction, susceptibilité, réceptivité, affinité, c'est-à-dire de la propension à percevoir ou non, à prendre positivement ou négativement l'expérience sensible que l'on a des choses. Si les relations sociales - celles qui se font et celles qui se défont - peuvent modeler nos perceptions, et changer l'image d'une donnée de connaissance, comme l'éducation physique, c'est parce qu'elles définissent le domaine de nos expériences et forment le cadre même où s'engendrent nos capacités perceptives et évaluatives.

De l'image de l'éducation physique, nous remontons à l'image de nous mêmes comme sujets sociaux faisant et défaisant l'objet que nous prenions, au premier abord, comme une entité extérieure et se faisant elle-même (avec le vieux démon des «logiques internes »). Ce changement de direction du regard, c'est celui auquel nous convient les sciences sociales, ici appliquées à des matériaux historiques. 
La leçon de gymnastique scolaire d'après Demény.

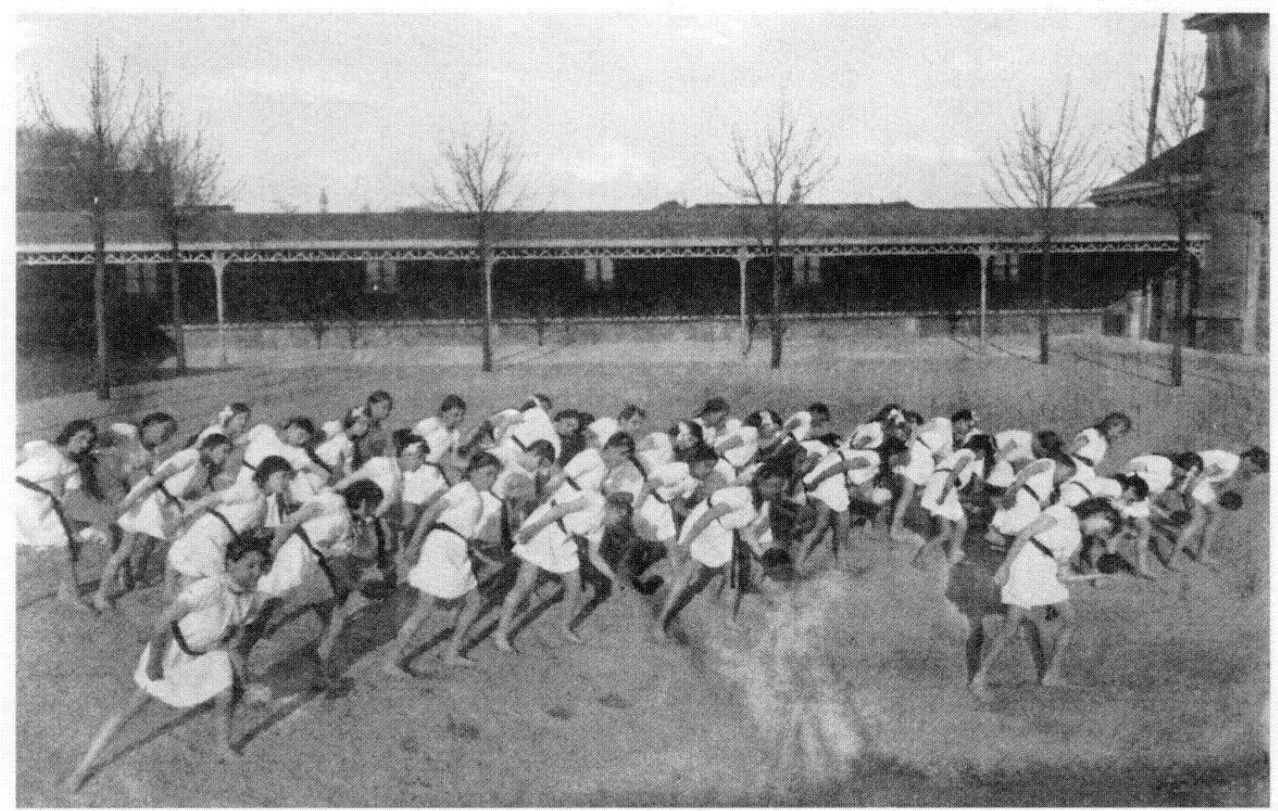

Une leçon donnée dans une école de filles de la ville de Reims, d'après une photographie communiquée par la Revue «L'Éducation Physique ». Académie des Sports et Comité National des Sports, Encyclopédie des Sports, Tome I, Paris, Librairie de France, 1924.

\section{BIBLIOGRAPHIE}

\section{Congrès}

Ministère du Commerce, de l'Industrie et des Colonies, 1889, Congrès International pour la propagation des exercices physiques dans l'éducation, Exposition Universelle de 1889, Procès-verbaux, Paris, impr. de l'Exposition, p.15.

$2^{e}$ Congrès national de l'Éducation physique, 1893, tenu à Bordeaux, Bordeaux, Gounouilhou. Congrès International, 1894 : discours de Pierre de Coubertin, in L'idée olympique. Discours et essais, Lausanne, éd. Internationales Olympia, 1966.

\section{Publications}

$1885-1896$

BONNEFONT G., Les exercices du corps, Paris, Jouvet et C $C^{\text {ie }} 1890$.

COMITÉ DES EXERCICES PHYSIQUES DANS L'ÉDUCATION, Manuel des jeux scolaires et des exercices athlétiques, Paris, Delalain, 1889. 
COUVREUR E., Les exercices du corps. Le développement de la force et de l'adresse. Étude scientifique. Paris, J.B. Baillière et fils, 1890.

DUPUY L.E., Le mouvement et les exercices physiques, Paris, J.B. Baillière et fils, 1893.

LAGRANGE F., L'hygiène de l'exercice chez les enfants et les jeunes gens, Paris, F. Alcan, 1890.

LAGRANGE F., De l'exercice chez les adultes, Paris, F. Alcan, 1891.

LAGRANGE F., La réforme de l'éducation physique, in La Revue des Deux Mondes, t. IV, Paris, 1892, 338-374.

LAGRANGE F., La médication par l'exercice, Paris, F. Alcan. MAREY E., Le mouvement, Paris, G. Masson, 1894, 1894.

MONIN E., La santé par l'exercice et les agents physiques, préf. de Ph. Daryl (alias P. Grousset), Paris, O. Doin, 1889.

SÉHÉ D., Strehly G., Manuel des exercices physiques à l'usage des écoles primaires. Exercices et nouveaux jeux, Paris, éd. Delagrave, 1890.

Années 1920 et 1930

HÉBERT G., Le sport contre l'éducation physique, Paris, Vuibert, 1925.

LAFARGE A., NAYRAC J.P., Traité de pédagogie de l'E.P. théorique et pratique, conçu selon les données de la biologie, de la psychophysiologie et de l'expérience, Paris, Hachette, 1934.

LOISEL E., Les bases psychologiques de l'E.P., Paris, Nathan, 1935.

TISSIÉ P., L'éducation physique et la race : santé, travail, longévité, Paris, Flammarion, 1919. VAN SCHAGEN K.H., Le rôle de l'éducation physique dans le développement de la personnalité, Paris, P.U.F., 1933.

Années 1955-1965

Archives Nationales, série F 44 bis 0eunesse et Sports) : F 44 bis 2539 (Haut Comité des Sports, Conseil National de l'Education physique) : 2540 (commissions formées au sein du HCS) : 2541 (commission de la doctrine).

Premier Ministre, Haut Comité des Sports, 1965, Essai de doctrine du sport, Paris, impr. Nationale.

\section{Études historiques et critiques}

ANDRIEU G. (éd.), Journées d'étude : L'éducation physique et le sport en France de 1913 à 1936 et les influences étrangères, Nanterre, Université Paris X, centre de Recherches en STAPS, 1989. ARNAUD P. et al., Le corps en mouvement. Précurseurs et pionniers de d'éducation physique, Toulouse, Privat, 1981.

ARNAUD P., Camy J., dir., La naissance du mouvement sportif associatif en France, Lyon, P.U.L, 1986.

ARNAUD P., Le Militaire, l'Écolier, le Gymnaste. Naissance de l'Éducation physique en France. 1869-1889, (préface de M. Agulhon), Lyon, Presses Universitaires de Lyon, 1991. BERNARD M., VIGARELLO G., POCIELLO C, « Itinéraire d'un concept », in Esprit, mai 1975, Paris, 704-723.

BOURDIEU P. La Distinction. Critique sociale du jugement, Paris, Minuit, 1979. BOURDIEU P., Le sens pratique, Paris, éd. Minuit, 1980.

BRUNER J., Acts of Meaning, 1990 : tr. fr. Car la culture donne forme à l'esprit. De la révolution cognitive à la psychologie culturelle, tr. fr. Paris, éd. Eshel, 1991. 
DEFRANCE J., l'excellence corporelle. La formation des activités physiques et sportives modernes. 1770-1914, Paris, revue STAPS : Rennes, Presses Universitaires de Rennes, 1987. DEFRANCE J., « La signification culturelle de l'Hébertisme. Etude de sociologie de la culture des années 1920 et 1930 ", in Sciences et Techniques des Activités Physiques et Sportives (Revue Francophone), n 31, mai 1993, Grenoble, PUG et AFRAPS, 47-63.

DEFRANCE J., « Le Sport contre l'Éducation physique et sa publication (en 1925) », Éducation physique et Sport, $\mathrm{n}^{\circ} 242$, Paris, EPS, 1993, 41-44.

DEFRANCEJ. « L'autonomisation du champ sportif. 1890-1970 », in Sociologie et Société (àparaître). FURET F., OZOUF J., Lire et écrire. L'alphabétisation des français de Calvin à Jules Ferry, Paris, éd. Minuit, 1977.

LE ROUX J., «Les premières sociétés françaises de sports athlétiques », in Pociello, dir., 1985.

MAUSS M., Oeuvres, tome 2, Représentations collectives et diversité des civilisations, présenté par V. KARADY, Paris, éd. Minuit, 1969.

OSTROW J.M., Social Sensitivity. A Study of Habit and Expérience, New-York, State University of NewYork Press, 1990.

POCIELLO G, Une tentative de rationalisation scientifique de l'éducation physique. Marev et Demény, Châtenay-Malabry, ENSEP, 1972.

POCIELLO G, dir., Travaux et Recherches en E.P.S., $n^{\circ} 8$, spécial Histoire sociale des pratiques sportives, 1985.

PROCHASSON G, Les années électriques. 1880-1910, Paris, La Découverte, 1991. SPIVAK M., Éducation Physique, Sport et Nationalisme en France du Second Empire au Front Populaire : un Aspect original de la Défense Nationale, Paris, Thèse d'État, Université Paris I, tome 3, 1983. THIBAULT J., Sport et Éducation physique (1870-1970), Paris, Vrin, 1972.

\section{NOTES}

1. En 1889 , une assemblée qui fédère sportifs et «éducateurs physiques » donne la prééminence aux seconds : voir les vœux émis par le congrès ; compte-rendu p. 13-14.

2. Au sein du Secrétariat d'État, des hauts fonctionnaires font la synthèse du document et en donnent une appréciation à l'usage du politique, appréciation pour le moins réservée : une note interne (signée: Grospeillet) indique que «la doctrine... apparaît comme un ensemble d'idées générales s'appliquant à tous les domaines du sport, sans qu'y apparaîsse... une ligne directrice ». A.N. F 44 bis / 2541.

3. Lequel ? Voir son rôle dans les commissions préparatoires des I.O. de 1962 et 1967 : A.N. F 44 bis / 2539.

4. L'État ne peut "l'aider (le CNEP) qu'à la condition qu'il ne sorte pas de son rôle qui est de prôner et de diffuser les différentes méthodes d'EP, d'autres organismes ayant pour rôle de prôner et de diffuser les autres activités, notamment l'initiation sportive, les sports et les activités de plein-air ». A.N. même cote.

5. Par exemple, dans une revue d'éducation physique scolaire, où elle fait déjà figure d'instance du passé : cf. "Pour un manifeste mondial de l'éducation physique ", Éducation Physique et Sports, $n^{\circ} 103,1970$, p. 15. 


\section{RÉSUMÉS}

L'analyse du champ de l'EP est habituellement effectuée sur la base de la constitution de liens sociaux. Au contraire, ici, l'application des sciences sociales, à des matériaux historiques constitués par l'histoire des rapports entre le champ de l'éducation physique, et celui notamment du sport, montre que l'image de ces champs se recompose et s'autonomise. Cette autonomisation est largement tributaire de la dislocation et de la rupture des liens qui rattachaient cette institution à d'autres institutions, telles que l'institution sportive ou militaire.

Analysis of the field of Physical Education is generally made on the basis of the constitution of social links/inter-relationships. On the contrary, here, the application of the social sciences, to historical artefacts/data brought together by the history of the relationships between the field of physical education and notably that of sports, shows that the image of these fields becomes redefined and autonomous. This process of becoming autonomous is to a large extent dependent upon the shake-up and the rupture of the inter-relationships by which this institution was linked to others, such as the institution of sports or to that of the military.

\section{INDEX}

Mots-clés : histoire, Images de l'éducation physique, lien social, représentations sociales, sociologie

Keywords : History, Image symbolic of physical éducation, Social inter-relationship, Social représentations, Sociology

\section{AUTEUR}

\section{JACQUES DEFRANCE}

Maître de conférences en S.T.A.P.S., université de Paris XI Orsay 